\title{
Autopodios de Guanacos en Sitios Arqueológicos: Equifinalidad entre lo Palatable y lo Preservado
}

\author{
Autopodials of Guanacos from Archaeological Sites: Equifinality Between Palatable and \\ Preserved
}

Laura Miotti ${ }^{\mathrm{y}}$ Laura Marchionni ${ }^{\mathrm{i}}$

\begin{abstract}
RESUMEN
La frecuencia de restos de autopodios de guanacos (Lama guanicoe) en los sitios arqueológicos de Patagonia indica que estas unidades tienen un patrón de hallazgo sobredimensionado frente a otras partes anatómicas del esqueleto. Las respuestas habituales a dichas frecuencias se refieren a cuestiones tafonómicas o a patrones de trozamiento y transporte diferencial. Por lo tanto, estas unidades de bajo retorno económico y alto índice de supervivencia, plantean un problema de equifinalidad. Aquí se discuten estas hipótesis a la luz del análisis comparativo de conjuntos zooarqueológicos de la Meseta Central de Santa Cruz, el que, sumado a la información etnográfica y de composición química de las grasas de artiodáctilos, amplía las interpretaciones zooarqueológicas, ayudando a minimizar la equifinalidad.
\end{abstract}

Palabras clave: Cazadores-Recolectores, Zooarqueología, Patagonia, Holoceno Medio.

\section{ABSTRACT}

The frequency of autopodial remains of guanacos (Lama guanicoe) in Patagonia archaeological sites indicates that these units present a finding oversized against other anatomical parts of the skeleton. The usual responses to these patterns are related to taphonomic issues or differential butchering and transport. Therefore, these units with low economic return and high survival rate present an equifinality problem. These hypotheses are discussed in light of the comparative analysis of sets zooarchaeological Central Plateau of Santa Cruz, which, combined with ethnographic and chemical composition of fats of artiodactyls extend zooarchaeological interpretations helping to minimize equifinality.

Key words: Hunter-Gatherers, Zooarchaeology, Patagonia, Mid Holocene.

\footnotetext{
i CONICET/División Arqueología, Facultad de Ciencias Naturales y Museo- UNLP, Paseo del Bosque s/nº, 1900, La Plata, Argentina. Correo-e: laura.miotti2@gmail.com; lau_marchionni@yahoo.com.ar
} 


\section{INTRODUCCIÓN}

En sitios arqueológicos de cazadoresrecolectores la sobredimensión de elementos del autopodio de ungulados de mediano y gran tamaño conduce a un problema de equifinalidad ya que su presencia no permite en primera instancia definir si corresponde a un transporte y descarte diferencial de partes anatómicas, o bien a huesos que, por mejores condiciones de preservación, quedaron sobrerrepresentados frente a otras unidades esqueletarias Estas y otras preguntas referidas al mismo problema surgieron en los pioneros trabajos de Binford al estudiar la variabilidad de partes anatómicas de caribúes (Rangifer tarandus) en contextos Nunamiut (Binford 1978). Desde esta perspectiva y con el trasfondo de la teoría del forrajeamiento óptimo, el problema radica en responder por qué unidades anatómicas de bajo retorno económico aparecen altamente representadas en sitios donde se llevaron a cabo diferentes actividades.

En Patagonia, el recurso principal de las sociedades cazadoras-recolectoras desde el Pleistoceno final fue el guanaco (Lama guanicoe), a diferencia de los caribúes del Ártico que son el recurso principal de los esquimales. Ambos herbívoros tienen rendimientos de carne y grasa diferentes, siendo más magra la carne de los guanacos (Borrero 1990, Lyman 1994). Sin embargo, desde la información etnoarqueológica y etnográfica, tanto en las sociedades del Ártico como en las de Patagonia austral, existen puntos de convergencia en el uso de dichos ungulados, los cuales tienen características etológicas similares, como la de ser animales gregarios y de amplia distribución en cada una de las dos áreas.

La equifinalidad referida a unidades anatómicas de guanacos se revela a partir de huesos que tienen una frecuencia moderada a alta, una densidad mineral ósea (DMO) alta y son de bajo retorno económico (Borrero 1990, Elkin 1995). En esta categoría entran los elementos del autopodio. Frente a esta recurrencia, se hace difícil determinar si tal representación es resultado de la selección y el transporte humano diferencial de los animales, o si dichas acumulaciones se deben a procesos tafonómicos.

Si bien algunos autores consideran, a partir de la realización de estudios actualísticos, que la
DMO puede cuestionarse como factor importante de reducción de los contextos zooarqueológicos (González et al. 2012), el problema de la equifinalidad continúa vigente. Como se discutirá más adelante, si bien los argumentos tafonómicos y de anatomía económica son útiles, han subestimado el aporte de estos elementos óseos en un marco de procesamiento para la obtención de nutrientes indispensables. Asimismo, fue minimizado su potencial de transporte y almacenamiento como "botiquín de emergencia" en lugares temporarios como detallan Yacobaccio et al. (1998) para pastores puneños. Por lo tanto, es importante incorporar otras líneas de evidencia que aporten nueva información para reducir el problema. Entre ellas, resulta relevante el trabajo experimental de Morin (2007) sobre la relación química de las diferentes grasas de grandes mamíferos terrestres y la palatabilidad humana, cuya aplicación a casos arqueológicos del Holoceno tardío de Patagonia fue realizada por Rindel (2009;20 I 3).Tanto la información etnoarqueológica, etnográfica y actualística sobre química de las grasas, ayudan a percibir desde otro ángulo las distintas estrategias de uso de las presas. A partir de este planteo nos proponemos analizar la representación de los autopodios de guanaco en tres contextos arqueológicos y en contextos etnográficos y etnoarqueológicos para abordar con otra mirada el problema. Por lo tanto, los objetivos de este trabajo son comparar y evaluar regionalmente la evidencia arqueológica de elementos del autopodio de sitios arqueológicos del Holoceno medio en la Meseta Central de Santa Cruz; utilizar los marcos de referencia de índices de grasa y carne, siguiendo los lineamientos de Binford (1978), Borrero (1990) y Morin (2007) y aportar información relevante, destacando las potencialidades de estas unidades anatómicas, más allá de la preservación.

\section{EL POTENCIAL DE LOS AUTOPODIOS EN LA TOMA DE DECISIONES}

La capacidad de retorno energético de los autopodios de guanaco es baja comparada con la de otras unidades como el estilopodio, cuyo poder calórico (carne y grasa) es superior (Borrero 1990). Considerando las diferentes partes que conforman el autopodio: basipodio, metapodio y acropodio, aquí nos referiremos principalmente al segundo segmento. La médula de los metapodios contiene grasas no 
saturadas (líquidas) que según estudios bioquímicos, tiene mejores cualidades para el consumo que las grasas saturadas (duras). Esta característica de los huesos largos de las porciones distales de las patas se debe a la mayor proporción de ácidos oleicos, los cuales son de más fácil digestión. Las implicancias de esta cualidad son, por un lado, que no es necesario someter el hueso a la cocción para consumir su contenido medular, de esta manera su ingesta puede ser directa, y aún así, su sabor y contenido alimenticio es muy bueno (Morin 2007, Rindel 2009). En segundo lugar, la fluidez de estos ácidos oleicos le confiere una mejor palatabilidad (Garvey 2010, Morin 2007).

Los metapodios, si no se agrietan, poseen, además, la propiedad de ser excelentes contenedores herméticos que mantienen por más de un año el buen estado de la médula para su consumo. Esta característica, es aprovechada por los pastores andinos como una estrategia de grasa diferida por medio de su almacenaje en las viviendas de verano en los campos altos de pastoreo (Yacobaccio et al. 1998).

Por último, es importante destacar que algunos pobladores tehuelche de Santa Cruz refieren a los metapodios como excelentes soportes para la confección de instrumentos "Pati: ...también sirven para hacer el punzón para clavar en los toldos..." (Aguerre 2000:116). Por lo cual, su frecuencia mediana a alta en los sitios podría deberse a su potencial como parte de cadenas operativas de instrumentos óseos (Miotti y Marchionni 2013). En los sitios aquí analizados fueron registrados instrumentos confeccionados sobre metapodios de camélidos con alto grado de formatización para la transición Pleistoceno-Holoceno y para el Holoceno medio, tanto en AEP-I como en Cueva Maripe. Además, en ambos sitios ha sido registrada la presencia de diáfisis de metapodio de guanaco modificadas por uso, cuya mayor representación se observa en los conjuntos del Holoceno medio de Cueva Maripe (Marchionni 2013). Un detalle cuali y cuantitativo de estos artefactos se consigna en Miotti (1998) y en Miotti y Marchionni (2013). Más allá de los sitios analizados en este trabajo, otros autores resaltan la existencia de un patrón de uso de los metapodios como retocadores blandos en distintos sitios de Patagonia (De Nigris 2004, Scheinsohn y Lucero 2006).
En suma, la información arqueológica y actualística avala que, en cazadores-recolectores así como en pastores, el consumo de estas unidades es materia de preferencia tanto en la dieta, como en la confección de instrumentos (Aguerre 2000, Binford 1978, Claraz 1988:59, 67, 105; Yacobaccio et al. 1998).

La alta DMO de los huesos del autopodio, confiere altas chances de preservación a estos elementos (Elkin 1995, Lyman 1994). La explicación más parsimoniosa es que su sobrerrepresentación se relaciona con una supervivencia mayor que la de otros huesos; sin embargo, una discusión crítica acerca de las variabilidad intraespecífica de la DMO (principalmente etaria) relativiza dicho potencial predictivo a esta interpretación (González et al. 2012). Estos autores encuentran gran variabilidad en los valores de DMO entre individuos de una misma especie así como dentro de la misma clase de edad. Los resultados obtenidos en su experimentación, considerando el desarrollo ontogenético de los guanacos, no les permiten hacer predicciones de grano fino acerca de la densidad en huesos de individuos de edades diferentes, y por consiguiente, no permite predecir las posibilidades de supervivencia de diferentes grupos de edad en conjuntos arqueológicos.

Todas estas potencialidades, tanto de mayor supervivencia como de ser un recurso importante para la vida de cazadores y pastores, son atributos destacables para convertir su registro arqueológico en un problema de equifinalidad.

\section{MATERIALESY MÉTODOS}

Se utilizaron los contextos arqueológicos correspondientes al Holoceno medio (ca. 7.5 - 3 ka AP) de la Meseta Central de Santa Cruz: Cueva 3 de Los Toldos, AEP-I de Piedra Museo y Cueva Maripe del Cañadón La Primavera (Figura I). Durante ese lapso estos sitios fueron ocupados como espacios domésticos por cazadores-recolectores (Marchionni et al. 2012, Miotti 1998, 2006; Miotti y Marchionni 20II). En éstos, como en la mayoría de los sitios patagónicos con cronologías similares, el guanaco ha sido la principal especie aprovechada. Asimismo, en ellos es recurrente la presencia mediana a alta de unidades anatómicas del esqueleto apendicular distal; este registro constituye un patrón de 
representatividad anatómica en el área (De Nigris 2004, Marchionni 2013, Mengoni Goñalons 1999, Miotti 1998, Rindel 2009, entre otros).

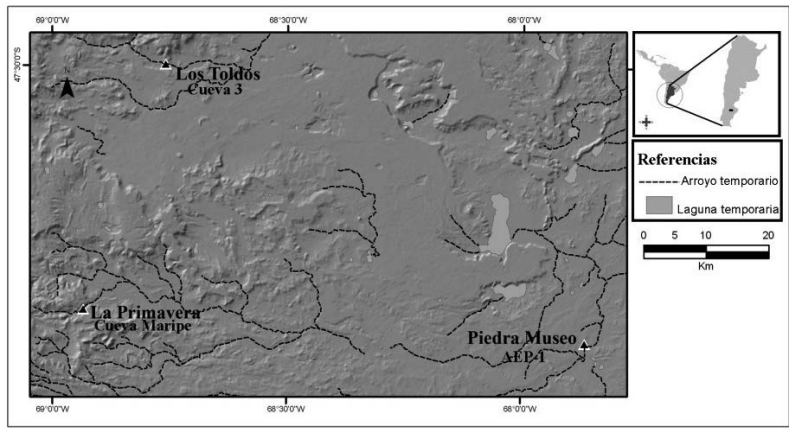

Figura 1: Mapa con la ubicación geográfica de los sitios arqueológicos considerados en el texto.

Figure 1: Map showing geographic location of archaeological sites considered in the text.

Estos conjuntos fueron seleccionados por brindar calidades similares de información. En todos ellos se consignan la cantidad y variedad de unidades anatómicas de guanaco siguiendo la misma metodología, resultando de ello unidades analíticas del mismo tenor

Los estudios tafonómicos, de procesamiento de las presas y de manufactura de instrumentos óseos para los tres contextos fueron intensamente detallados en trabajos previos (Marchionni 2013, Marchionni et al. 2012, Miotti 1998, Miotti y Marchionni 2009, 20II, 2013). Los resultados indican tendencias hacia una buena preservación de los conjuntos, una baja a nula incidencia de acción de carnívoros y roedores, una alta frecuencia de marcas de procesamiento entre las que se destacan principalmente para los metapodios, huellas de corte y fracturas longitudinales con atributos asociados. Asimismo, como se expresó más arriba, en estos conjuntos se registra una alta frecuencia de artefactos formales e informales confeccionados en metápodos (Miotti y Marchionni 2013).

La representación de partes anatómicas y su potencial de transporte humano fue evaluada agrupando los especímenes óseos según las grandes regiones esqueletarias, siguiendo los lineamientos de Mengoni Goñalons (1999) con algunas modificaciones. Las zonas consideradas son: cabeza, cervicales y tronco para el esqueleto axial; estilopodio, zeugopodio, autopodio y cinturas, para el esqueleto apendicular (Lyman 1994:87-89).

El análisis comparativo interconjunto se realizó sobre la base de las frecuencias de NISP total para cada región y los valores derivados de MNE, MAU y \%MAU. Para estos últimos tres estimadores se consideró sólo el valor máximo exhibido entre los distintos elementos óseos que conforman cada región. La representación relativa de las diferentes regiones se realizó a partir de la frecuencia máxima de \%MAU discriminando, en el caso del esqueleto apendicular, las porciones anteriores y posteriores. Este análisis cobra relevancia ya que el metabolismo de las grasas medulares varía entre estas porciones, dependiendo del estado nutricional de los animales (Morin 2007).

La evaluación de las distintas estrategias de transporte y uso de las presas se realizó en base a las clases propuestas por Lyman (1994) a partir de las correlaciones estadísticas entre los valores de \%MAU, DMO (Elkin 1995) y \%MGUI (Borrero 1990).

Para ampliar la comparación de transporte y uso de los autopodios se utilizó analógicamente información etnoarqueológica y etnográfica, lo que permitió generar expectativas de presencia/ausencia en el registro arqueológico.

\section{RESULTADOS}

\section{Zooarqueológicos}

Sobre un total de 2452 especímenes óseos de guanaco para los componentes arqueofaunísticos del Holoceno medio, 907 (37\%), corresponden a huesos del autopodio (Figura 2a).

El análisis de elementos óseos por grandes regiones anatómicas indica que los huesos del autopodio tienen representación significativa, si bien las frecuencias son variables en cada conjunto. Los menores porcentajes de las patas distales se encuentran en la capa 3 de la Cámara Norte de Cueva Maripe (Figura 2). Sin embargo, para el mismo rango temporal, tanto en la Cámara Sur de la misma cueva como en AEP-I, el \%MAU de autopodios alcanza $100 \%$, mientras que en la Cueva 3 de Los Toldos, alcanza el 62,8\% (Figura $2 b$ ). 
Las correlaciones estadísticas entre \%MAU, DMO y \%MGUI varían en los conjuntos resultando en diferentes modelos de estrategias de supervivencia y transporte (Tabla I). Según Lyman (I994), Los Toldos correspondería a una estrategia "bulk or gourmet utility" y AEP-I a "Reverse utility, lag or ravaged". En Cueva Maripe el panorama es más complejo, mientras que en Cámara Norte el conjunto de Capa 4 corresponde a "class 5 " o equifinalidad, el de Capa 3, de la misma cámara, y el de la Unidad Analítica 3 (UA3), de Cámara Sur, corresponden a "lag o ravaged" (Marchionni 20I3). En tal sentido, lo que observamos al comparar estos conjuntos con porcentajes de autopodios que, en todos los casos superan el $60 \%$ del NISP, es que su alta frecuencia en los sitios define por sí solo el tipo de acarreo y formas de descarte de los animales. Sin embargo, considerando la significativa producción de artefactos confeccionados en metápodos de guanacos de todos los contextos analizados, se podría reducir el valor interpretativo asignado a las categorías de equifinalidad, y proponer asignar una importancia mayor al transporte intencional de estas unidades anatómicas en estos sitios. Esta intencionalidad de transporte a los sitios de actividades múltiples podría deberse a las potencialidades que ofrecen estas unidades como: a) elementos clave para atar los animales completos como parte de una estrategia de traslado de presas o como partes que viajan unidas al cuero usado como bolsa y que lleva dentro algunas partes de las pesas (con mayores proporciones de carne y grasa) al lugar doméstico para su consumo; b) otra posibilidad, en función de la significativa presencia de instrumentos confeccionados sobre metápodos, es que dichos huesos hayan sido transportados intencionalmente a los sitios, donde, se les podría haber extendido la vida útil transformándolos, luego del consumo de su alimento (médula), en instrumentos, como pueden ser los retocadores, punzones, etc.

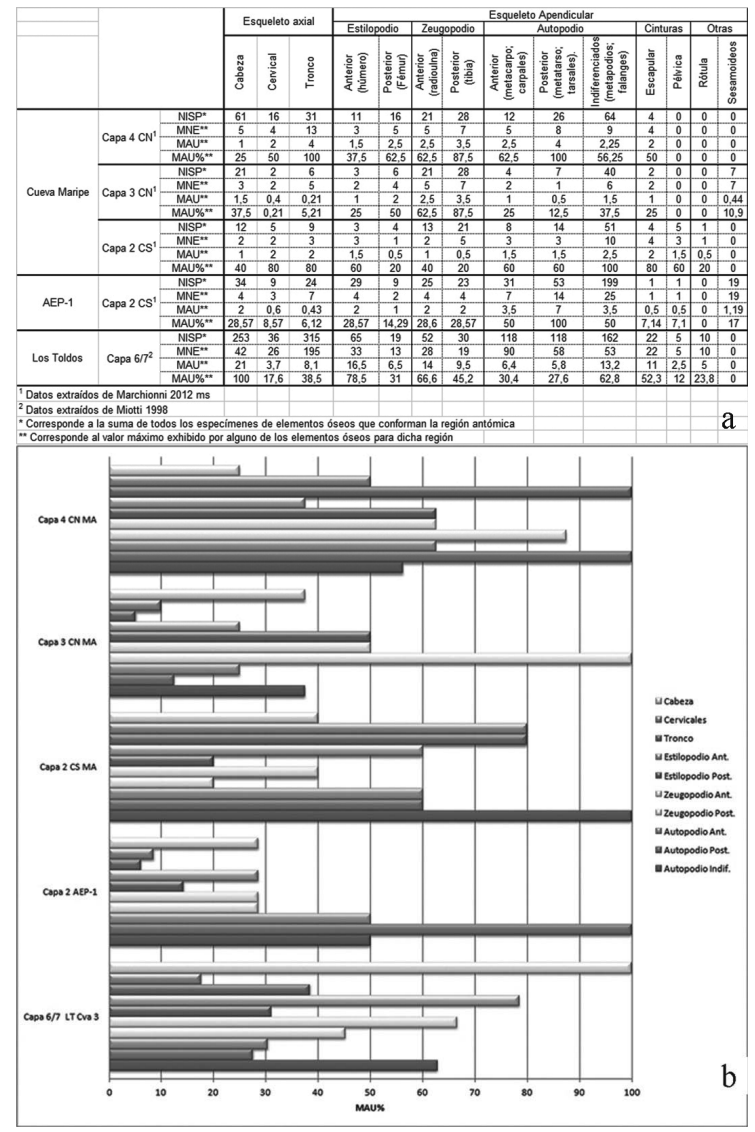

Figura 2: a) Tabla de abundancia relativa de unidades anatómicas de guanaco expresada en NISP, MNE, MAU y \%MAU; b) Histograma de abundancia de unidades anatómicas de guanaco expresada en \%MAU y agrupadas por grandes regiones anatómicas.

Figure 2: a) Table shows relative abundance of guanaco anatomical units. Expressed in NISP, MAU, and \%MAU; b) Histogram shows relative abundance of guanaco anatomical units expresed in \%MAU and clustered by big anatomical regions. 


\section{Etnográficos y etnoarqueológicos}

Los modelos Selk'nam pedestre y Tehuelche ecuestre varían como en otros grupos cazadoresrecolectores en las formas de trozamiento $y$ transporte de las presas, según cantidad de cazadores y distancias a los consumidores (Bettinger 1991, Binford 1978, Kelly 1995) pero también por otras razones, referidas a la palatabilidad, estado de gordura de los animales y el transporte de partes de las cuales se dará luego del consumo alimenticio, un uso posterior. Por ejemplo, si la caza es de tipo solitaria en el bosque, como la practicada a finales del otoño/ invierno por los Selk'nam (Gallardo 1910), o bien colectiva como la Tehuelche de momentos ecuestres, generalmente en la estepa (Claraz 1988, Musters, 1964). Por otro lado, la estación del año y el estado de gordura de los animales en el momento de la cacería incide en las formas de trozamiento y transporte de las presas (Miotti 1998, Miotti y Marchionni 2009). Una tercera fuente de variabilidad, es la distancia entre el sitio de matanza y el campamento familiar, que puede alterar incluso, las formas de movilidad de los grupos.

\begin{tabular}{|c|c|c|c|c|c|}
\hline & \multicolumn{3}{|c|}{ Maripe } & AEP-I & LT \\
\hline & Capa 4 CN & Capa 3 CN & UA3 CS & Capa 2 & Cueva 3 \\
\hline \%MAU-DMO & $\begin{array}{c}\text { No significativo } \\
(r=0,35 ; p= \\
0,051)\end{array}$ & $\begin{array}{l}\text { Moderada, positiva } \\
\text { y significativa } \\
(r=0,52 ; p= \\
0,0009)\end{array}$ & $\begin{array}{l}\text { Baja a moderada, } \\
\text { positiva y significativa } \\
(r=0,34 ; p=0,03)\end{array}$ & $\begin{array}{c}\text { Moderada, positiva y } \\
\text { significativa } \\
(r=0,43 ; p=0,006)\end{array}$ & $\begin{array}{c}\text { No significativo } \\
(r=0,082 ; p=0,62)\end{array}$ \\
\hline \%MAU-MGUI & $\begin{array}{c}\text { No Significativo } \\
\left(\begin{array}{c}r=-0,18 ; p= \\
0,33)\end{array}\right.\end{array}$ & $\begin{array}{c}\text { No significativo } \\
(r=-0,027 ; p= \\
0,87)\end{array}$ & $\begin{array}{c}\text { No significativo } \\
(r=0,09 ; p=0,57)\end{array}$ & $\begin{array}{c}\text { Moderada a alta, } \\
\text { negativa y significativa } \\
(r=-0,63 ; p=0,00002)\end{array}$ & $\begin{array}{c}\text { Baja a moderada, } \\
\text { positiva y significativa } \\
(r=0,36 ; p=0,026)\end{array}$ \\
\hline
\end{tabular}

Tabla 1: Correlaciones estadísticas

Table 1: Statistical correlations

El transporte de unidades de bajo rendimiento cárneo y/o graso, desde los lugares de matanza, hacia las bases residenciales puede estar indicando las preferencias de calidades de grasas de mejor sabor y digestibilidad, así como su valor como materia prima para la producción de instrumentos óseos. Esto es similar en el caso de pastores de Puna, en donde estas unidades se encuentran tanto en las bases residenciales (casas grandes del valle) como en los puestos de pastoreo estacional de altura, habitados sólo en la estación estival por las pastoras (Yacobaccio et al. 1998).
A partir de esta información, en la figura 3 se sintetizan las expectativas teóricas de hallazgos en sitios arqueológicos. Por lo tanto, a la luz de esta información actualística es esperable hallar arqueológicamente tanto en bases residenciales, como en sitios de actividades especiales (de matanza y trozamiento primario) elementos del autopodio de guanacos. Esta expectativa excede, por lo tanto, las causas de preservación mayor que tienen estos elementos y revaloriza la selección humana de los mismas para su transporte preferencial. 


\begin{tabular}{|c|c|c|c|c|c|c|c|c|}
\hline \multirow{3}{*}{\multicolumn{2}{|c|}{ UNIDADES }} & \multicolumn{3}{|c|}{ Tehuelche ecuestre (Muster 1964) } & \multicolumn{2}{|c|}{$\begin{array}{l}\text { Selk'nam pedestre } \\
\text { (Gallardo 1910) }\end{array}$} & \multicolumn{2}{|c|}{$\begin{array}{c}\text { Pastores de Susques (Yacobaccio } \\
\text { et al. 1998) }\end{array}$} \\
\hline & & \multicolumn{2}{|c|}{ Estación de Caza } & \multirow{2}{*}{$\begin{array}{c}\text { Base Residencial } \\
\text { Procesamiento, } \\
\text { reparto, consumo y } \\
\text { descarte }\end{array}$} & \multirow[b]{2}{*}{$\begin{array}{c}\text { Estación de } \\
\text { Caza }\end{array}$} & \multirow[b]{2}{*}{$\begin{array}{l}\text { Base } \\
\text { residencial }\end{array}$} & \multirow[b]{2}{*}{$\begin{array}{c}\text { Residencia } \\
\text { estación de lluvia o } \\
\text { puesto de altura }\end{array}$} & \multirow[b]{2}{*}{$\begin{array}{c}\text { Casa familiar en } \\
\text { zona baja del } \\
\text { valle }\end{array}$} \\
\hline & & $\begin{array}{c}\text { Matanza y } \\
\text { Procesamiento } \\
1^{\circ}\end{array}$ & \begin{tabular}{|c|} 
Matanza y \\
Procesamiento \\
$1^{\circ}$ y $2^{\circ}$
\end{tabular} & & & & & \\
\hline \multirow{3}{*}{ Esqueleto Axial } & Cabeza & $A$ & \begin{tabular}{|c|}
$P$ \\
\end{tabular} & $P$ & $\bar{A}$ & $P$ & $\bar{A}$ & $\bar{P}$ \\
\hline & Cervical & A & $\mathrm{P}$ & $\mathrm{P}$ & A & $\mathrm{P}$ & A & $\mathrm{P}$ \\
\hline & Tronco & A & AVP & $P$ & A & $P$ & A & $\mathrm{P}$ \\
\hline \multirow{7}{*}{ Esqueleto Apendicular } & Estilopodio Ant. & A & A & $P$ & A & $\mathrm{P}$ & A & $\mathrm{P}$ \\
\hline & Estilopodio Post. & $P$ & A & A & A & $P$ & A & $\mathrm{P}$ \\
\hline & Zeugopodio Ant. & $P$ eventual & A & $\mathrm{P}$ & $P$ eventual & $P$ eventual & A & $\mathrm{P}$ \\
\hline & Zeugopodio Post. & $P$ eventual & A & $P$ & $P$ eventual & $P$ eventual & A & $\mathrm{P}$ \\
\hline & \begin{tabular}{|l|} 
Autopodio Ant. \\
\end{tabular} & $P$ eventual & A & $\mathrm{P}$ & $P$ eventual & $P$ eventual & $\mathrm{P}$ & $\mathrm{P}$ \\
\hline & Autopodio Post. & $P$ eventual & A & $\mathrm{P}$ & $P$ eventual & $P$ eventual & $\mathrm{P}$ & $\mathrm{P}$ \\
\hline & Autopodio Indif. & $P$ eventual & A & $\mathrm{P}$ & $P$ eventual & $P$ eventual & $\mathrm{P}$ & $\mathrm{P}$ \\
\hline
\end{tabular}

Figura 3: Modelos etnoarqueológico y etnográfico expresados por presencia (P) y ausencia (A) de las diferentes unidades anatómicas registradas entre los cazadores-recolectores de la Patagonia y pastores andinos. Sus implicaciones arqueológicas se desprenden de las expectativas teóricas acerca de la movilidad de los cazadores-recolectores.

Figure 3: Ethnoarchaeological and ethnographic models display presence $(P)$ and absence $(A)$ of different anatomical units recorded among Patagonian hunter-gatherers and Andean herders. Their archaeological implications result from their theoretical expectations about huntergatherer mobilities'.

Es importante considerar que dentro de estas "preferencias" se engloban una serie de estrategias de transporte por parte de los cazadores recolectores, quienes privilegian los costos de traslado de animales completos o ya trozados según las distancias entre el lugar de caza y el de consumo; la cantidad de cazadores intervinientes en las partidas de caza y los costos de procesamiento de los animales en el lugar de matanza o en el campamento base (Bettinger 1991, Bettinger y Baumhoff 1982, Binford 1991, Kelly 1995; entre muchos otros). Pero también deben contemplarse las cuestiones de reparto social de las presas ya trozadas, entre todos los integrantes del grupo (Marshall 1993, Gallardo 1910) y las cuestiones de tabúes sobre el consumo en el campamento o fuera de él de ciertas presas (Politis y Saunders 2004).

\section{CONSIDERACIONES FINALES}

Se ha presentado información relevante tanto arqueológica como etnográfica para considerar que la abundancia de autopodios en sitios arqueológicos puede responder a intereses culturales como la calidad de su grasa, más fina y palatable, el consumo diferido y su importancia para la elaboración de instrumentos. Por lo tanto, su presencia puede darse tanto en estaciones de caza como en bases residenciales, situación que se observa en el registro arqueológico del área (Figura 2) y en la información actualística (Figura 3). Sin embargo, no se descarta que la destrucción tafonómica sea un factor más, pero no el más importante, para explicar la sobrerrepresentación de huesos del autopodio en los contextos arqueológicos.

La evidencia arqueológica y la analogía etnográfica, permiten sugerir la alta significación que tendrían los autopodios en los circuitos de transporte y consumo, almacenamiento de grasas y confección de instrumentos. Desde esta perspectiva social de la intencionalidad de transporte, la presencia de huesos del autopodio, que ingresan a las bases residenciales adjuntos a unidades de mayor retorno económico "schlepp effect" o por el efecto "the hunter' sewing kit" se vería relativizada (Lyman 1994).

La intencionalidad humana considerada aquí para interpretar las representaciones de autopodios en contextos zooarqueológicos, sean los mismos estaciones de caza o sitios residenciales, propone nuevas alternativas como son la preferencia por la mejor palatabilidad del contenido de estos huesos en grasas no saturadas y para la confección de instrumentos óseos; las cuales amplían el rango de interpretaciones a las tradicionalmente formuladas hasta el momento y basadas principalmente en la potencialidad de preservación y retorno energético que conllevan estas unidades anatómicas. La analogía etnográfica y etnoarqueológica, 
ayudó a re-configurar patrones de consumo basados únicamente en los retornos calóricos de las distintas partes anatómicas, produciendo expectativas mayores respecto de las cualidades de preferencias del consumo de sus productos. En tal sentido lo pensamos como una clave para reflexionar sobre variables no sólo de retorno básico, sino sensoriales (gusto) y pragmáticas como el almacenamiento para consumos diferidos y producción de instrumentos.

\section{AGRADECIMIENTOS:}

Los coordinadores del II ELAZ propiciaron la presentación de este trabajo en Chile. Rocío Blanco y Enrique Terranova leyeron el manuscrito aportando valiosos comentarios. Esta investigación fue financiada por ANPCYT y FCNyM-UNLP con subsidios PICT I 552 y PI 556.

\section{BIBLIOGRAFÍA}

Aguerre, A. 2000. Las Vidas de Pati en la toldería Tehuelche del Río Pinturas y el después. Facultad de Filosofía y Letras-UBA, Buenos Aires.

Bettinger, R. L., 1991. Hunter-Gatherers: Archaeological and Evolutionary Theory. Plenum Press, New York/London.

Bettinger, R. y M, Baumhoff, 1982. "The Numic spread: Great Basin cultures in competition". American Antiquity 47(3):485-503.

Binford, L. 1978. Nunamiut Etnoachaeology. Academic Press, New York.

--1991. "A corporate caribou hunt. Documenting the archaeology of the past lifeways". Arctic Journal 33 (I):33-43.

Borrero, L. 1990. "Fuego-Patagonian bone assemblages and the problem of communal guanaco hunting". En Hunters of the recent past, editado por Davis, L. \& B. Reeves, Pp. 373-399. One world archaeology 15, Unwin Hyman, London.

Claraz, J. 1988. Diario de Viaje de la Exploración al Chubut1865-I866. Marymar, Buenos Aires.

De Nigris, M. 2004. El Consumo en Grupos Cazadoresrecolectores. Un Ejemplo Zooarqueológico de Patagonia Meridional. Sociedad Argentina de Antropología, Colección Tesis doctorales, Buenos Aires, Argentina.

Elkin, D. 1995. "Volume density of South America camelids skeletal parts". International Journal of Osteoarchaeology 5: 29-37.

Gallardo, C. R. 1910. Los Onas. CABAUT y Cía., Buenos Aires.

Garvey, J. 2010. Bennett's Wallaby marrow quality vs. quantity: evaluating human decision-making and seasonal occupation in late Pleistocene Tasmania. Abstract of the oral and poster presentation, XI ICAZ, pp. I 37. Francia, Paris.

González, M.; M. Álvarez, A. Massigoge, M. Gutiérrez y C. Kaufmann. 2012. "Bone differential survivorship and ontogenic development in guanaco (Lama guanicoe)". International Journal of Osteoarchaeology 22: 523-536.

Kelly, R. L.1995. The Foraging Spectrum. Smithsonian Institution Press, Washington y London.
Lyman, R. 1994. Vertebrate Taphonomy. Cambridge University Press, UK.

Marchionni, L. 2013. Comparación de las distintas historias tafonómicas en conjuntos zooarqueológicos provenientes de la Meseta Central de Santa Cruz. Tesis doctoral inédita, FCNyM- UNLP.

Marchionni, L.; B. Mosquera y E. García Añino. 2012. "Analysis of zooarchaelogical and taphonomical variability from Maripe Cave site, Santa Cruz province, argentinian Patagonia”. Quaternary International 278: I2-2I.

Marshall, F. 1993. "Food Sharing and the Faunal Record". En From Bones to Behavior: Ethnoarchaeological and Experimental Contributions to the Interpretation of Faunal Remains, editado por J. Hudson, pp. 228-46. Center for Archaeological Investigations, Occasional Paper 21, Southern Illinois University at Carbondale.

Mengoni Goñalons, G. 1999. Cazadores de Guanacos de la Estepa Patagónica. Sociedad Argentina de Antropología, Buenos Aires.

Miotti, L. 1998. Zooarqueología de la Meseta Central y Costa de la Provincia de Santa Cruz: Un Enfoque de las Estrategias Adaptativas Aborígenes y los Paleoambientes. Museo Municipal de Historia Natural de San Rafael, Mendoza.

.-2006. "Paisajes Domésticos y Sagrados desde La Arqueología De Los Cazadores-Recolectores En El Macizo Del Deseado, Provincia De Santa Cruz". Cazadores Recolectores Del Cono Sur, Revista De Arqueología I: I3- 42.

Miotti, L. y L. Marchionni. 2009. "Procesando huesos: entre la Etnografía y la Arqueología”. En Arqueología de la Patagonia. Una mirada desde el último confin, editado por M. Salemme, F. Santiago, M. Álvarez, E. Piana, M. Vázquez y E. Mansur, Tomo II, Pp. 787-798. Utopías, Ushuaia,Tierra del Fuego.

--120 II."The study of archaeofauna at middle Holocene in AEP-I rockshelter, Santa Cruz, Argentina: Taphonomic implications". Quaternary International 245: I 48-I58.

---2013. Beyond Stones:"Bone as raw material for Tools in the Central plateau of Santa Cruz,Argentinean Patagonia”. En From these bare bones: Raw materials and the study of worked osseous objects, editado por A. Choyke y S. O'Connor, pp. I I6-I26. Oxbow, London.

Morín, E. 2007. "Fat composition, skeletal part selection, and Nunamiut decision- making”. Journal of Archaeological Science 34:69-82.

Musters G. C. 1964. Vida entre los Patagones. Un año de Excursiones por Tierras no Frecuentadas desde el Estrecho de Magallanes hasta el Río Negro. Ediciones Solar-Hachette, Bs. As.

Politis, G. y N. Saunders. 2004. "Archaeological Correlates of Ideological Activity: Food Taboos and Spirit-animals in an Amazonian Hunter-gatherer Society". En Consuming Passions and Patterns of Consumption, editado por P. Miracle y N. Milner, pp. I I3130, Cambridge Monographs.

Rindel, D. 2009. Arqueología de Momentos Tardíos en el Noroeste de la Provincia de Santa Cruz (Argentina): una Perspectiva Faunistica. Tesis Doctoral inédita, FFyL- UBA.

Rindel, D. 2013. "Marcos de referencia y frecuencia de partes esqueletarias de guanacos en sitios de Patagonia meridional: el caso de los índices de médula e índice de médula insaturada". En Tendencias teórico-metodológicas y casos de estudio en la arqueología de la Patagonia, compilado por A. Zangrando, R. Barberena;A. Gil; G. Neme; M. Giardina; L. Luna; C. Otaola; S. Paulides; L. Salgán y A.Tivoli, Pp. 5I 5-522. Museo de Historia Natural, San Rafael, Mendoza.

Scheinsohn, V. y M. Lucero. 2006. Explotación de Materias primas óseas en el sur del continente: el caso de Cerro Casa de Piedra. Disponible on-line en BoneCommons http://www. alexandriaarchive.org/bonecommons/items/show/465

Yacobaccio, H.; Madero, C. y M. Malmierca. 1998. Etnoarqueología de pastores surandinos. Grupo Zooarqueología de camélidos (eds.) 119 pp. Buenos Aires. 